\title{
Dietary Vitamin E Supplementation Does Not Inhibit Candida albicans Intestinal Translocation in Rats
}

\author{
Márcia Varella Morandi, Roberto Martinez and Hélio VANnUCCHI* \\ Faculty of Medicine of Ribeirão Preto, University of São Paulo, \\ 14049-900 Ribeirão Preto, SP, Brazil
}

(Received July 11, 1998)

\begin{abstract}
Summary Candida albicans translocation was determined in rats receiving a normal or vitamin E-supplemented and deficient diet submitted to mesenteric ischemia and reperfusion (MIR). The antioxidant effect of vitamin $\mathrm{E}$ on lipid peroxidation was also assessed. The animals were divided into six groups submitted to different diets for $30 \mathrm{~d}$. Groups N, NI, NC and NIC were submitted to a normal diet and used as controls, and groups VITE and DEFE received a vitamin E-supplemented and vitamin E-deficient diet, respectively. Groups NIC, VITE and DEFE were submitted to MIR, inoculated with Candida albicans and sacrificed $24 \mathrm{~h}$ after the surgical procedure. The antioxidant effect of vitamin E was determined in the liver and gut mucosa using the TBARS method. Candida albicans translocation was assessed in lymph node, liver and kidney specimens. The results showed that lipid peroxidation was lower $(p<0.05)$ in the vitamin E-supplemented group. However, vitamin E supplementation did not protect the rats against Candida albicans translocation (the translocation in the Group VITE was $100 \%$ for lymph nodes).

Key Words intestinal translocation, free radical, vitamin E, Candida albicans, mesenteric ischemia
\end{abstract}

Some conditions are essential for the mucosal barrier to exert its protective function against microorganisms in the intestinal lumen. These functions are the maintenance of intestinal microflora, efficient host immunologic system and intact mucosal epithelium in the small intestine. When one or more factors are altered, intestinal translocation of microorganisms is favored (1). This is characterized by microorganisms passing through the intestinal wall to extraintestinal sites such as mesenteric lymph nodes and appearing in viable conditions in the circulation and various organs. Today, this mechanism is thought to be responsible for situations of sepsis without a defined primary focus that mainly occur in patients with severe impairment of general conditions, often leading to multiple organ failure $(2,3)$.

* To whom correspondence should be addressed. E-mail: hvannucc@fmrp.usp.br 
When a tissue is exposed to ischemia, a sequence of chemical reactions is observed that may lead to cell dysfunction and tissue injury (4). This injury is caused by different chemical reactions that occur after oxygenated blood returns to the ischemic tissue. These reactions resulting from reperfusion involve cytotoxic oxidants derived from molecular oxygen (i.e., reactive oxygen metabolites or free radicals) $(5,6)$. One of the most important mechanisms and probably the initial source of free oxygen radicals during ischemia and later reperfusion in the intestine is the hypoxanthinexanthine oxidase system, with the intestine being a site highly rich in xanthine dehydrogenase (7).

Among the lesions caused by free radicals in practically all types of organic matter, particularly important is the involvement of lipid biomembranes (8). Lipid peroxidation is generally started by the attack of several reactive species, with the occurrence of a chain reaction and of lipid peroxide accumulation on the membrane. Lipid peroxides destabilize the membranes and make them permeable to ions (9-11).

The ability of vitamin $\mathrm{E}$ to protect cell membrane lipids against oxidative damage has been fully elucidated $(12,13)$. Vitamin $\mathrm{E}$ acts by supplying hydrogen atoms to the cell membranes, preventing the chain reaction from propagating through lipid membranes. $\alpha$-Tocopherol inhibits lipid peroxidation by reacting with lipid peroxide $(6)$. The objective of the present study was to determine the antioxidant effect of vitamin $\mathrm{E}$ in the neutralization of free radicals produced by mesenteric ischemia and reperfusion and its consequent protection against Candida albicans translocation.

\section{MATERIALS AND METHODS}

A total of 59 adult male Wistar rats weighing on average $148 \mathrm{~g}$ were divided into six groups: Group 1 (control), N, rats on a normal diet not submitted to Candida albicans inoculation or ischemia; Group 2 (control), NI, rats on a normal diet not submitted to Candida albicans inoculation but submitted to mesenteric ischemia and reperfusion; Group 3 (control), NC, rats on a normal diet submitted to Candida albicans inoculation but not to ischemia; Group 4 (control), NIC, rats on a normal diet submitted to Candida albicans inoculation, mesenteric ischemia and reperfusion; Group 5 (test), VITE, rats on a diet supplemented with vitamin E submitted to Candida albicans inoculation, mesenteric ischemia and reperfusion; Group 6 (test), DEFE, rats on a vitamin E-deficient diet submitted to Candida albicans inoculation, mesenteric ischemia and reperfusion.

The diets were prepared according to the AOAC, 1995 (14). Supplementation of the diet for Group 5 was vitamin E provided in an amount 20 times higher $(0.156 \mathrm{~g} / 100 \mathrm{~g}$ of diet $)$ than recommended for normal rat diet $(0.009 \mathrm{~g} / 100 \mathrm{~g}$ of diet $)$. The rats received the diets described above for $30 \mathrm{~d}$. On day zero of the experiment, the rats received a Candida albicans inoculum (the concentration of inoculum was $1.0 \times 10^{10}$ colony-forming units of Candida albicans $/ \mathrm{ml}$ of inoculum) by gavage, and $60 \mathrm{~min}$ later, were submitted to laparotomy for the induction of ischemia 
Table 1. Plasma level of vitamin E.

\begin{tabular}{ccccccc}
\hline & \multicolumn{6}{c}{ Groups } \\
\cline { 2 - 7 } & $\begin{array}{c}\mathrm{N} \\
(n=8)\end{array}$ & $\begin{array}{c}\mathrm{NI} \\
(n=8)\end{array}$ & $\begin{array}{c}\mathrm{NC} \\
(n=8)\end{array}$ & $\begin{array}{c}\text { NIC } \\
(n=9)\end{array}$ & $\begin{array}{c}\text { VITE } \\
(n=13)\end{array}$ & $\begin{array}{c}\text { DEFE } \\
(n=13)\end{array}$ \\
\hline Vitamin E & $10.17^{\mathrm{ab}}$ & $18.16^{\mathrm{a}}$ & $17.64^{\mathrm{ab}}$ & $9.89^{\mathrm{ab}}$ & $28.16^{\mathrm{a}}$ & $1.73^{\mathrm{b}}$ \\
& \pm 9.16 & \pm 11.16 & \pm 14.18 & \pm 8.19 & \pm 15.75 & \pm 1.62 \\
\hline
\end{tabular}

$\mathrm{N}$, group on a normal diet not submitted to ischemia or Candida albicans inoculation. NI, group on a normal diet submitted to ischemia but not to Candida albicans inoculation. $\mathrm{NC}$, group on a normal diet not submitted to ischemia but submitted Candida albicans inoculation. NIC, group on a normal diet submitted to ischemia and Candida albicans inoculation. VITE, group on a diet supplemented with vitamin $\mathrm{E}$ and submitted to ischemia and Candida albicans inoculation. DEFE, group on a vitamin E-deficient diet submitted to ischemia and Candida albicans inoculation. Means followed by different letters are significantly different $(p<0.05)$.

Values represent mean $\pm \mathrm{SD}(\mu \mathrm{mol} / \mathrm{L})$.

induced by clamping the mesenteric superior artery. Reperfusion was carried out $40 \mathrm{~min}$ later. Twenty-four hours after surgery, the animals were anesthetised with ether and sacrificed. All mesenteric lymph nodes of the distal ileum, a liver fragment and the left kidney were removed, weighed and processed for Candida albicans culturing. The positives cultures were determined by counting the number of colony-forming units of Candida albicans/g of tissue. For the assessment of lipid peroxidation, we determined thiobarbituric acid reactive substances (TBARS) in the liver and mucosa by the method of Uchiyama and Mihara (15). Plasma $\alpha$-tocopherol was determined by HPLC (16)

This experiment was done in accordance with the Recommendations of the Helsinki Declaration [Med Clin (Barc), 1988; 91(18): 702-703], the protocol was approved by the Animal Experiment Ethical Committee of the School of Medicine of the University of São Paulo.

\section{RESULTS}

Table 1 presents the plasma concentrations of vitamin $\mathrm{E}$ in the groups. The Kruskal-Wallis test showed a significant difference between group DEFE and groups NI and VITE.

The TBARS concentrations (mean $\pm \mathrm{SD}$ ) in the intestinal mucosa and liver are presented in Table 2. The Kruskal-Wallis test showed a significant difference in the intestinal mucosa between groups $\mathrm{N}$ and DEFE, NI and VITE, NIC and VITE, and VITE and DEFE. In the liver, there was a significant difference between group DEFE and the remaining groups.

Table 3 presents the percentages of positive Candida albicans cultures for mesenteric lymph nodes, liver and kidney. The chi-square test shows that there was 
Table 2. Thiobarbituric acid reactive substances (TBARS) in the intestinal mucosa and liver.

\begin{tabular}{lcccccc}
\hline \multirow{2}{*}{ TBARS } & \multicolumn{6}{c}{ Groups } \\
\cline { 2 - 7 } & $\begin{array}{c}\mathrm{N} \\
(n=8)\end{array}$ & $\begin{array}{c}\mathrm{NI} \\
(n=8)\end{array}$ & $\begin{array}{c}\mathrm{NC} \\
(n=8)\end{array}$ & $\begin{array}{c}\text { NIC } \\
(n=9)\end{array}$ & $\begin{array}{c}\text { VITE } \\
(n=13)\end{array}$ & $\begin{array}{c}\text { DEFE } \\
(n=13)\end{array}$ \\
\hline Intestinal & $0.355^{\mathrm{ab}}$ & $0.692^{\mathrm{bc}}$ & $0.397^{\mathrm{abc}}$ & $0.644^{\mathrm{bc}}$ & $0.294^{\mathrm{a}}$ & $0.776^{\mathrm{c}}$ \\
-mucosa & \pm 0.121 & \pm 0.233 & \pm 0.085 & \pm 0.309 & \pm 0.094 & \pm 0.319 \\
Liver & $0.546^{\mathrm{a}}$ & $0.819^{\mathrm{a}}$ & $0.787^{\mathrm{a}}$ & $1.329^{\mathrm{a}}$ & $0.599^{\mathrm{a}}$ & $3.029^{\mathrm{b}}$ \\
& \pm 0.163 & \pm 0.329 & \pm 0.487 & \pm 0.502 & \pm 0.125 & \pm 0.536 \\
\hline
\end{tabular}

$\mathrm{N}$, group on a normal diet not submitted to ischemia or Candida albicans inoculation. NI, group on a normal diet submitted to ischemia but not to Candida albicans inoculation. NC, group on a normal diet not submitted to ischemia but submitted to Candida albicans inoculation. NIC, group on a normal diet submitted to ischemia and Candida albicans inoculation. VITE, group on a diet supplemented with vitamin $\mathrm{E}$ and submitted to ischemia and Candida albicans inoculation. DEFE, group on a vitamin E-deficient diet submitted to ischemia and Candida albicans inoculation. Means followed by different letters are significantly different $(p<0.05)$.

Values represent mean $\pm \mathrm{SD}$ ( $\mathrm{nmol} / \mathrm{mg}$ protein).

Table 3. Median and statistical variability of the minimum and maximum values (min-max) of log colony-forming units (cfu) and percentage (\%) of positive Candida albicans cultures in mesenteric lymph nodes, liver and kidney.

\begin{tabular}{lcccccc}
\hline \multirow{2}{*}{ Translocation } & \multicolumn{6}{c}{ Groups } \\
\cline { 2 - 7 } & \begin{tabular}{c}
$\mathrm{N}$ \\
\cline { 2 - 7 }
\end{tabular} & $\begin{array}{c}\text { NI } \\
(n=8)\end{array}$ & $\begin{array}{c}\text { NC } \\
(n=8)\end{array}$ & $\begin{array}{c}\text { NIC } \\
(n=9)\end{array}$ & $\begin{array}{c}\text { VITE } \\
(n=13)\end{array}$ & $\begin{array}{c}\text { DEFE } \\
(n=13)\end{array}$ \\
\hline Lymph nodes (cfu) & 0 & 0 & 2.3 & 3.7 & 3.5 & 2.6 \\
min-max & $(0-0)$ & $(0-0)$ & $(0-2.7)$ & $(0-4.4)$ & $(2.8-4.8)$ & $(0-4.4)$ \\
$(\%)$ & 0 & 0 & 75 & 89 & 100 & 84.6 \\
Liver (cfu) & 0 & 0 & 0 & 2.7 & 2.4 & 0 \\
min-max & $(0-0)$ & $(0-0)$ & $(0-2.9)$ & $(0-5.5)$ & $(0-4.8)$ & $(0-2.89)$ \\
$(\%)$ & 0 & 0 & 25 & 78 & 69 & 43 \\
Kidney (cfu) & 0 & 0 & 0 & 1.7 & 0 & 0 \\
min-max & $(0-0)$ & $(0-0)$ & $(0-0)$ & $(0-4.5)$ & $(0-4.2)$ & $(0-2.4)$ \\
$(\%)$ & 0 & 0 & $0^{\mathrm{a}}$ & $55.5^{\mathrm{b}}$ & 30.7 & 38.4 \\
\hline
\end{tabular}

$\mathrm{N}$, group on a normal diet not submitted to ischemia or Candida albicans inoculation. NI, group on a normal diet submitted to ischemia but not to Candida albicans inoculation. $\mathrm{NC}$, group on a normal diet not submitted to ischemia but submitted to Candida albicans inoculation. NIC, group on a normal diet submitted to ischemia and Candida albicans inoculation. VITE, group on a diet supplemented with vitamin $\mathrm{E}$ and submitted to ischemia and Candida albicans inoculation. DEFE, group on a vitamin E-deficient diet submitted to ischemia and Candida albicans inoculation. Means followed by different letters are significantly different $(p<0.05)$. 
no significant difference between the groups submitted to inoculation $(p>0.05)$. There was a significant difference $(p<0.05)$ in the percentage of positive kidney cultures between NIC and NC.

\section{DISCUSSION}

The symptoms of vitamin E deficiency are considered to be the result of damage caused by free radicals (12). Lindley et al (17) showed that after 16 weeks of exposure to deficient and supplemented diets, rats in the deficient group had undetectable plasma $\alpha$-tocopherol concentrations, whereas the supplemented group had concentrations of $34.5 \pm 1.4 \mu \mathrm{mol} / \mathrm{L}$. In the present study, Table 1 shows that DEF animals had significantly lower plasma vitamin E levels $(1.73 \pm 1.62 \mu \mathrm{mol} / \mathrm{L})$ as compared to the remaining groups that received diets containing normal or supplemented vitamin $\mathrm{E}$ amounts $(28.16 \pm 15.75 \mu \mathrm{mol} / \mathrm{L})$.

The present study also demonstrated an increase in TBARS levels in the intestinal mucosa, with a significant difference between the vitamin E-deficient DEFE group and the $\mathrm{N}$ group (i.e., a control group that was not submitted to surgery or Candida albicans inoculation) and the vitamin E-supplemented VITE group, whose TBARS concentration was lower than that in the DEFE group. There was a significant difference between group VITE (supplemented), group NI (submitted to ischemia only and receiving a normal diet) and group NIC (submitted to a normal diet, ischemia and Candida albicans inoculation). The TBARS concentration was lower in group VITE than in groups NI and NIC, and was similar to the concentration found in group $\mathrm{N}$ (Table 2). The increased TBARS production in the intestinal mucosa of rats submitted to ischemia alone or ischemia and reperfusion shows that lipid peroxidation plays an important role in the production of damage to the intestinal mucosa (18).

TBARS production in the liver was significantly higher in group DEFE as compared to groups N, NI, NC, NIC and VITE (Table 2). Other investigators have shown that rats fed normal and vitamin E-deficient diets present increased TBARS concentrations in the liver (19-21). Another report (22) showed that, after $90 \mathrm{~min}$ of hepatic ischemia and reperfusion from 0 to $60 \mathrm{~min}$ in rats injected intraperitoneally with a placebo or vitamin $\mathrm{E}$, the MDA values in liver tissue remained relatively stable during the period of ischemia in both groups. After reperfusion, there was an increased production of MDA in the placebo group, whereas in the vitamin E-treated group, this MDA increase was inhibited.

In the present study, the Candida albicans translocation was determined by counting the log number of colony-forming units (cfu) in positive cultures. There was not a significant difference in terms of the percentage of positive cultures detected in the organs from the different groups inoculated (Table 3), however, there was a significant difference $(p<0.05)$ between the NC and NIC control groups in the kidney measurements. The mechanism of disequilibrium in the intestinal flora caused by the oral inoculation of Candida albicans was probably responsible for 
the translocation of the microorganisms observed in the NC control group where the translocation to mesenteric lymph nodes and liver occurred at rates of 75 and $25 \%$, respectively. No fungal colonies were detected in the kidney of this group, which was not submitted to surgery or anesthesia. The process of ischemia involves the alteration of intestinal permeability, and can provoke higher microorganism translocation to systemic circulation, as observed in groups NIC, VITE and DEFE, where translocation to all the studied tissues occurred.

The model of mesenteric ischemia and reperfusion used here reflects the hemodynamic and metabolic changes occurring in situations of hypermetabolism such as hipovolemic shock, postoperative stress and trauma. In animals submitted to ischemia and reperfusion, Candida albicans translocation was observed in all organs studied. For the mesenteric lymph nodes, the percentages of Candida albicans positive cultures were $89 \%$ for the NIC control group, $100 \%$ for the VITE experimental group and $84.6 \%$ for the DEFE experimental group. The percentages of positive cultures for the liver were $78 \%$ for the NIC control group, $69 \%$ for the VITE experimental group and $43 \%$ for the DEFE group, indicating dissemination through portal circulation. In the renal parenchyma, which reflects the translocation disseminated through systemic circulation, the percentages of positive cultures were $55.5 \%$ for group NIC, $30.7 \%$ for group VITE and $38.4 \%$ for group DEFE. When we compared the log colony-forming units of Candida albicans in the groups submitted to inoculation, we did not observe a significant difference in translocation, although translocation was higher in the groups submitted to ischemia (Table 3). In a previous study from our laboratory (23) using rats on a zinc-deficient diet and later submitted to mesenteric ischemia and reperfusion, we demonstrated that translocation was higher for animals submitted to ischemia than in animals that were not submitted to the procedure; demonstrating a greater magnitude of the phenomenon caused by ischemia-reperfusion.

All of these results indicate the production of free radicals during ischemia and mainly during reperfusion, probably damaging the intestinal mucosa and causing increased translocation.

Immunodeficiency plays an extremely important role in the occurrence of translocation and propagation of the microorganism to distant locations. In studies in which the immune response is not significantly altered, translocation is limited to the lymph nodes for a short period of time. These lymph nodes should normally destroy a large part of the microorganisms that reach them, but failure of the immunologic system prevents elimination of the microorganisms, permitting them to colonize the lymph nodes and to remain viable (24).

In the present study, we did not observe an effect of vitamin $E$ on the process of Candida albicans translocation since no significant difference in the log colony-forming units (cfu) was observed between the groups submitted to fungal inoculation. However, there was a significant reduction of lipid peroxidation in the intestinal mucosa and in the liver of group VITE as compared to groups DEFE and NIC. It is possible that macrophage activation was altered due to vitamin $\mathrm{E}$ 
supplementation, with the consequent inhibition of superoxide anion. It should be pointed out that the superoxide anion produced by leucocytes is an important oxygen radical for bacterial death. Since vitamin $\mathrm{E}$ is an antioxidant, the mechanism of bacterial killing by leucocytes can be inhibited by the administration of high doses of this vitamin. Some studies have demonstrated that very high plasma levels of vitamin $\mathrm{E}$ may predispose premature infants to infection, including necrotic enterocolitis and sepsis $(25,26)$. Similarly, there are several studies showing the longer survival of animals submitted to treatment with $\alpha$-tocopherol. Some investigators have demonstrated improved survival in experimental models of endotoxemia after treatment with $\alpha$-tocopherol, and others have reported a reduction of lipid peroxidation in hepatocyte membranes, with improved cell energy and increased survival of animals exposed to endotoxins $(27,28)$. Endogenous oxidation reactions are essential for the maintenance of the normal biochemistry of living organisms and are especially important in terms of the killing action of leucocytes against microbes (i.e., in terms of the mechanism of host defense in infectious diseases). However, reactive oxygen intermediates may damage normal tissues unless they are under the control of an antioxidant (29).

Thus, in biological systems there is an absolute necessity for the control of the reactions that release free radicals and their reactive intermediary compounds by antioxidants or scavengers. This control, however, should be balanced, since complete elimination of these free radicals from the cell may cause its destruction. It is necessary to think about oxidative stress and antioxidants as a system in which failure of either element in the balance may result in some type of disorder (29). On this basis, Krinsky (30) defines biological antioxidants as "compounds that protect biological systems against the harmful effects of processes or reactions that may cause excessive oxidation."

Antioxidants are clearly important in cell regulation but the appropriate balance between oxidative processes and antioxidants still needs to be defined. Biological antioxidants do not act separately but as part of a complex system $(13,31-33)$.

Thus, on the basis of the results obtained, we may conclude that vitamin $\mathrm{E}$ supplementation led to lower lipid peroxidation in the intestinal mucosa but did not protect the animal against Candida albicans translocation.

We wish to thank Mrs. M. S. M. Bernardes, Mr. A. Verceze, Mr. M. R. Arantes, Mr. A. Jardim, Mr. A. A. Jordão and Mrs. M. Borges for their excellent technical assistance.

\section{REFERENCES}

1) Deitch EA. 1993. Multiple organ failure. Adv Surg 26: 333-356.

2) Deitch EA, Berg R. 1987. Bacterial translocation from the gut: a mechanism of infection. $J$ Burn Care Rehabil 8: 475-482.

3) Wells CL, Maddaus MA, Simmons RL. 1990. Evidence for the translocation of enterococcus faecalis across the mouse intestinal tract. J Infect Dis 62: 82-90.

4) Mitsudo S, Brand LJ. 1992. Pathology of intestinal ischemia. Surg Clin North Am 72: 
43-63.

5) Wong S, Knight JA, Hopfer SM, Zaharia O, Leach CN Jr, Sunderman FW. 1987. Lipoperoxides in plasma as measured by liquid chromatographic separation of malondialdehide-thiobarbituric acid adduct. Clin Chem 33: 214-220.

6) Halliwell B, Gutteridge JMC. 1989. Free Radicals in Biology and Medicine, 2a. ed. Clarendon Press, Oxford.

7) Grisham MB, Hernandez LA, Granger DN. 1986. Xantine oxidase and neutrophil infiltration in intestinal ischemia. Am J Physiol 251: 567-574.

8) Rice-Evans CA. 1994. Formation of free radicals and mechanisms of action in normal biochemical processes and pathological states. In: Free Radical Damage and Its Control (Rice-Evans CA, Burton RH, eds), p 129-151. Elsevier, Amsterdam.

9) Bjønrboe B, Bjønrboe GE, Drevon CA. 1990. Absorption, transport and distribution of vitamin E. J Nutr 120: 233-242.

10) Abbey M, Nestel PJ, Baghurst PA. 1991. Antioxidant vitamins and low-densitylipoprotein oxidation. Am J Clin Nutr 53: 201S-205S.

11) Jialal I, Grundy SD. 1992. Influence of antioxidant vitamins on LDL oxidation. Ann NY Acad Sci 669: 237-248.

12) Burton GW. 1994. Vitamin E: molecular and biological function. Proc Nutr Soc 53: 251-262.

13) Jacob RA. 1995. The integrated antioxidant system. Nutr Res 15: 755-766.

14) AOAC. 1995. Official Methods of Analytical Chemists of Association of Official Analytical Chemists, 12a ed, Washington.

15) Uchiyama M, Mihara M. 1978. Determination of malonaldehyde precursor in tissues by thiobarbituric acid test. Anal Biochem 86: 271-278.

16) Arnaud J, Blachieri FS, Kia D, Favier A. 1991. Simultaneous determination of retinol, alfa-tocopherol and beta-carotene in serum by isocratic high-performance liquid chromatography. J Chromatrogr 572: 103-116.

17) Lindley MA, Sampson MAG, Muller PPR, Milla PJ. 1994. Lipid peroxidation and electrogenic ion transport in the jejunum of the vitamin E-deficient rat. Gut 35: 34-39.

18) Yoshikawa T, Ueda S, Naito Y, Takahashi S, Oyamada H, Morita Y, Yoneta T, Kondo M. 1989. Role of oxygen-derived free radicals in gastric mucosal injury induced by ischemia or ischemia-reperfusion in rats. Free Rad Res Comms $7: 285-291$.

19) Lee H, Csallany AS. 1987. Measurement of free and bound malondialdehyde in vitamin E-deficient and supplemented rat liver tissues. Lipids 22: 104-107.

20) Lee H, Csallany AS. 1994. The influence of vitamin E and selenium on lipid peroxidation and aldehyde dehydrogenase activity in rat liver and tissue. Lipids 29: 345-350.

21) Vannucchi H, Morandi MVM, Iglesias AC, Jordão Jr AA, Chiarello PG. 1997. Effect of different dietary levels of vitamin E on lipid peroxidation in rats. Arch Latino-Am Nutr 47: 34-37.

22) Marubayashi S, Dohi K, Surgino K, Kawasaki T. 1989. The protective role of administered tochopherol against hepatic damage caused by ischemia-reperfusion or endotoxemia. Ann NY Acad Sci 570: 208-218.

23) Portari Filho PE, Iglesias AC, Martinez R, Vannucchi H. 1997. Zinc deficiency and intestinal translocation of Candida albicans in rats submitted to laparotomy and mesenteric ischemia. In: The Immune Consequences of Trauma, Shock and Sepsis. Mechanisms and Therapeutic Approaches (Faist E, ed), p 223-227. Manduzzi Editore, Bologna, Italy.

24) Wells CL, Maddaus MAW, Simmons RL. 1988. Proposed mechanisms for the translocation of intestinal bacteria. Rev Infect Dis 10: 958-979. 
25) Johnson L, Bowen FW, Abrasi S, Herrmann N, Weston M, Sacks L, Porat R, Stahl G, Peckham G, Delivoria-Papadoupoulos M, Quinn G, Schaffer D. 1985. Relationship of prolonged pharmacologic serum levels and necrotizing enterocolitis in infants with birth weight 1,500 grams or less. Pediatrics 75: 619-637.

26) Mino M. 1992. Clinical uses and abuses of vitamin E in children. Proc Soc Exp Biol Med 200: 266-270.

27) Ogilvie AC, Groeneveld ABJ, Straub JP, Thijs LG. 1991. Plasma lipid peroxides and antioxidants in human septic shock. Int Care Med 17: 40-44.

28) Powell RJ, Machiero GW, Rush BF, George D. 1991. Effect of oxygen-free radical scavengers on survival in sepsis. Am Surg 57: 86-89.

29) Keusch GT. 1993. Antioxidants in infection. J Nutr Sci Vitaminol 39: S23-S33.

30) Krinsky N. 1992. Mechanism of action of biological antioxidants. Proc Soc Exp Biol Med 200: 248-254.

31) Olson JA, Kobayashi S. 1992. Antioxidants in health and disease: Overview. Proc Soc Exp Biol Med 200: 245-247.

32) Packer L. 1992. Interactions among antioxidants in health and disease: Vitamin E and its redox cycle. Proc Soc Exp Biol Med 200: 271-276.

33) Halliwell B, Aeshbach R, Löliger J, Aruoma OI. 1995. The characterization of antioxidants. Food Chem Toxicol 33: 601-617. 